\title{
Use of fixation/fusion to treat lumbar spinal stenosis
}

\author{
Robert Heary, MD
}

Department of Neurosurgery, Rutgers University Medical Center, Newark, New Jersey

$\mathrm{T}$

HE authors present a paper that challenges many long-established principles in the treatment of lumbar spinal stenosis (LSS). ${ }^{1}$ They treated 70 consecutive patients with LSS over a 4-year period with posterior lumbar fixation and fusion-without performing neural decompressions - as the sole treatment modality. This concept is unique and new to the treatment of lumbar spine disorders.

In their study, the authors present a retrospective assessment of the treatment of 70 patients. They used a posterior fixation surgery with the Goel facet distraction spacer, which stabilized the facet joints, to facilitate posterior spinal fusions. Fusions were incorporated into the procedure but no neural decompressions were included in their surgeries. Interestingly, the levels treated were determined intraoperatively by the surgeons, using palpation, rather than by advanced neuroimaging studies. The results obtained in this study are dramatic and surpass any results reported by North American spine surgeons. The authors report that all patients were "very satisfied" with their outcomes. Furthermore, they describe " $100 \%$ symptom relief" in their cohort. In addition, the authors have reported that successful fusions occurred in all 70 patients treated. Throughout the 28-month mean follow-up, they did not observe the recurrence of symptoms in any case.

Interestingly, a similar method of treatment has been described by numerous Asia-Pacific-region spine surgeons for the treatment of cervical spondylotic myelopathy (CSM). In these novel surgical treatments, the spine surgeons used posterior stabilization and fusion, without decompression, for the treatment of CSM. Despite numerous presentations having been made at a number of spine specialty societies on this topic, in which the use of stabilization/fusion without decompression for CSM is described, we were not able to locate either peer-reviewed journal publications or published abstracts that specifically addressed this approach. It would appear that some of the current authors' methodology would be similar to that used by these surgeons from the Asia-Pacific region whose results in the treatment of cervical spine pathology have not included decompression of the neural elements. With that said, some North American spine surgeons have continued to express skepticism over the treatments that lack decompression for CSM, and this treatment has not yet achieved widespread acceptance in North America. A comprehensive effort to find these surgeries described in peer-reviewed journals was unsuccessful. It is possible that the principles espoused by these spine surgeons may be responsible for an increase in the number of posterior cervical spine surgeries in the United States and for the recognition of the importance of strong stabilization constructs and solid arthrodeses in achieving ideal clinical outcomes in CSM surgeries.

There are some aspects of the current paper presented by Goel et al. that are concerning. The potential for bias or conflict of interest is always present when the technique used to treat the patients diverges from the mainstream approaches, and when this technique uses a proprietary device invented by the lead author of the paper being presented. In order to counteract any potential criticism of this procedure, it would behoove the authors to either recruit other spine surgeons to help reproduce the results of their study model, and confirm or refute the results, or continue this treatment model themselves with independent evaluators who would determine the fusion status and the clinical outcomes separately from the group of treating physicians. Using the approach of assessing consecutive patients with acceptable follow-up, as was reported in this study, would afford the spine community the opportunity to make its own determinations of the value of this approach, which is a clear deviation from the traditional methods widely used in North America.

Dr. Goel and his associates have reported results that exceed by a great extent any outcomes reported after using 
traditional, mainstream principles of neural decompression in the treatment of LSS. As with any groundbreaking techniques that deviate strongly from the traditional approaches, the authors should be strongly encouraged to have their results corroborated independently, which is the best manner to gain widespread acceptance in the scientific community.

https://thejns.org/doi/abs/10.3171/2019.2.FOCUS19139

\section{References}

1. Goel A, Ranjan S, Shah A, Patil A, Vutha R: Lumbar canal stenosis: analyzing the role of stabilization and the futility of decompression as treatment. Neurosurg Focus 46(5):E7, 2019

\section{Disclosures}

The author reports no conflict of interest.

\section{Correspondence}

Robert Heary: heary@njms.rutgers.edu.

INCLUDE WHEN CITING

DOI: 10.3171/2019.2.FOCUS19139. 